Article

\title{
Tensile Creep Behavior of Quasi-Unidirectional E-Glass Fabric Reinforced Polypropylene Composite
}

\author{
Zhanyu Zhai ${ }^{1, *}$, Bingyan Jiang ${ }^{2}$ and Dietmar Drummer ${ }^{1}$ \\ 1 Institute of Polymer Technology, University Erlangen-Nuremberg, 91054 Erlangen, Germany; \\ dummer@lkt.uni-erlangen.de \\ 2 State Key Laboratory of High Performance and Complex Manufacturing, Central South University, \\ Lushan South Road 932, 410083 Changsha, China; jby@csu.edu.cn \\ * Correspondence: zhai@lkt.uni-erlangen.de; Tel.: +49-9131-852-9722
}

Received: 29 May 2018; Accepted: 10 June 2018; Published: 13 June 2018

\begin{abstract}
The present work addressed the creep behavior of quasi-unidirectional E-glass fabric reinforced polypropylene composites under off-axis tensile loading. A series of creep tests were performed on the composite at three different loading stress levels. The creep response of off-axis samples of quasi-unidirectional composites under a constant loading level can be clearly observed. A phenomenological viscoplasticity model was built for describing the creep behavior of the composite. To improve the accuracy of prediction, cyclic loading-unloading tests were adopted to determine the material constants in the model. The predicted results in terms of the strains after a load over a period of time were found to be satisfactory, compared with the experimental results. In addition, same failure mechanism was found in off-axis samples under quasi-static and creep loading cases.
\end{abstract}

Keywords: tensile creep; thermoplastic composites; viscoplasticity model

\section{Introduction}

Fiber reinforced thermoplastic composites (FRTCs) have been widely used in many applications. As the matrix for composites, thermoplastic polymers exhibit many advantages over thermosets, like short processing time and intrinsic recyclability [1]. The durability of FRPCs is required in their service life. The long service time in combination with the viscous properties in the thermoplastic polymers emphasize the requirement of the investigation of long-term behavior of FRTCs [2]. Creep behavior is one of the time-dependent responses of material, which is a continuous deformation under a constant load [3,4]. For advanced fiber reinforced polymer composites, fibers are usually assumed as linearly elastic and do not creep. Therefore, the creep behavior observed in composites originates from the matrix [5]. Polymers generally undergo significant creep behavior even at room temperature [6]. However, it is demonstrated that fiber reinforcement can limit the creep behavior of matrix in FRTCs $[7,8]$. In the past years, most of research on the creep behavior of FRTCs has mainly focused on discontinuous fiber reinforced thermoplastic composites (short fibers [7,9] and long fibers $[10,11])$. The experimental results showed that the creep behavior of composites is affected by the factors such as the creep behavior of matrix, the geometry, the distribution of reinforcement and the fiber-matrix interfacial properties.

So far, some investigations have carried on the creep behavior of continuous fiber reinforced thermoplastic composites (C-FRTCs) through experimental and numerical methods [2,4,12]. Liou and Teng [4] conducted a systematic investigation of the creep behavior of unidirectional (UD) carbon fiber/nylon 6 composites in the fiber direction as well as off-axis direction at three different temperatures. They found that the composite was linear viscoelastic if the off-axis angle was smaller than $30^{\circ}$, whereas, nonlinear viscoelastic behavior was observed when off-axis angle was increased 
and subjected to high stress level. The Findley power [13] was used to describe the viscoelastic creep behavior of composites. Brauner et al. [2] carried out creep tests on C-FRTCs with respect to both loading temperature and loading stress level. The extended Burgers model was used to predict the long-term viscoelastic creep behavior of composites.

On the other hand, some phenomenological viscoplasticity models were proposed in literatures [5,14-17] to predict both rate-dependent nonlinear behavior and creep behavior of polymeric composites. It should be noted that, in contrast to metals, polymeric composites do not have the plasticity mechanical behavior. However, the macro-mechanical constitutive models for polymeric composites use the terminology that is more familiar and originally developed in viscoplasticity theory [18]. The strain rate-dependent inelastic deformation including viscous and viscoelastic parts in polymeric composites are characterized by viscoplasticity. These proposed phenomenological viscoplasticity models were developed based on the one-parameter plasticity model proposed by Sun [19]. However, as illustrated by our previous work [20], the one-parameter plasticity model overestimates the plastic strain since the model fails to identify the contributors (i.e., damage and plasticity) to nonlinearity in composites. Thus, it leads to a less accurate prediction of creep strain, especially for high creep stress levels.

The objective of this work is characterizing the tensile creep behavior of quasi-unidirectional E-glass fabric reinforced polypropylene composites (quasi-UD E-glass/polypropylene composites) both experimentally and constitutively. For this purpose, tensile creep tests were firstly performed at three different loading stress levels on quasi-UD E-glass/polypropylene composites. By taking a similar approach with the viscoplasticty model proposed by Sun et al. [21], a phenomenological viscoplasticity model was developed for quasi-UD E-glass/polypropylene composites. In this model, the plastic strains of composites were determined through cyclic loading-unloading tensile tests. It was found that the predicted creep strains of this model were in good agreement with the experimental results. In addition, the failure mechanisms of composites under quasi-static loading and creep loading were also discussed.

\section{Modeling of Creep Behavior}

The strains of composites induced by a constant stress over a long period of time are composed of time-independent initial strains and time-dependent creep strains. In terms of the incremental formulation, it can be written as [5]

$$
d \varepsilon_{t}=d \varepsilon_{\text {in }}+d \varepsilon_{c}
$$

where $\varepsilon_{t}$ are the total strains over a long period of time $t$. The initial strains $\varepsilon_{i n}$ are assumed to occur instantaneously as the load is applied. $\varepsilon_{\mathcal{c}}$ are the creep strains.

For quasi-UD E-glass/polypropylene composite under a constant stress $\sigma_{0}$, the time-independent initial strain $\varepsilon_{i n}$ can be determined from a coupled damage-plasticity model proposed in our previous work [20]. According to the continuum damage mechanics (CDM) developed by Ladeveze [22], the material stiffness loss in composites is characterized by the damage variable. The emergence of permanent strains is described as plasticity theory. Thus, the axial strain increments of quasi-UD E-glass/polypropylene composites under quasi-static loading are composed of the elastic and plastic parts

$$
d \varepsilon_{x}=d \varepsilon_{x}^{e}+d \varepsilon_{x}^{p}=\frac{d \sigma_{x}}{(1-D) E_{x}}+d \varepsilon_{x}^{p}
$$

where $\varepsilon_{x}$ and $\sigma_{x}$ are axial strain and stress, respectively. $\varepsilon_{x}^{e}$ is the elastic strain, and $\varepsilon_{x}^{p}$ is the plastic strain. Subscript $x$ refers to the loading direction. $D$ is the damage variable. $E_{x}$ is the elastic modulus of virgin material.

Based on the associated flow rule, the incremental plastic strains $d \varepsilon_{i j}^{p}$ can be written as

$$
d \varepsilon_{i j}^{p}=\frac{\partial f}{\partial \sigma_{i j}} d \lambda
$$


where the stresses $\sigma_{i j}(i, j=1,2,3)$ refer to the principal material directions; 1-direction coincides with fiber direction; 2-direction is perpendicular to fiber direction; $f$ is the plastic potential function proposed by Cho [23] and $d \lambda$ is a proportionality factor.

Since there is no plastic deformation in the fiber direction of quasi-UD E-glass/polypropylene composite [24], the plastic potential function in the case of plane stress state can be simplified as

$$
f=\sqrt{a_{2} \sigma_{22}^{2}+\sigma_{12}^{2}}+b_{2} \sigma_{22}
$$

where $a_{2}$ and $b_{2}$ are the plasticity parameters.

The effective stress $\bar{\sigma}$. is defined as

$$
\bar{\sigma}=f
$$

Following [20], it can be shown that

$$
d \lambda=d \bar{\varepsilon}_{p}
$$

in which $\bar{\varepsilon}_{p}$ is the effective plastic strain.

For orthotropic composites subjected to off-axis tension, the effective stress and effective plastic strain are related to the applied axial stress and plastic strain as

$$
\begin{gathered}
\bar{\sigma}=h(\theta) \sigma_{x} \\
\bar{\varepsilon}_{p}=\frac{\varepsilon_{x}^{p}}{h(\theta)}
\end{gathered}
$$

with

$$
h(\theta)=\sqrt{a_{2} \sin ^{4} \theta+\sin ^{2} \theta \cos ^{2} \theta}+b_{2}
$$

Plastic strain $\varepsilon_{x}^{p}$ under the applied stress $\sigma_{x}$ can be directly obtained after the axial loading stress vanishes upon unloading, as shown in Figure 1. The master effective stress-effective plastic strain relationship can be fitted approximately with a power law [19]

$$
\bar{\varepsilon}_{p}=A(\bar{\sigma})^{n}
$$

where $A$ and $n$ are parameters to be determined from experimental results.

Finally, a coupled damage-plasticity model for quasi-UD E-glass/polypropylene composite under quasi-static loading is determined as

$$
\varepsilon_{x}=\frac{\sigma_{x}}{E_{0} \exp \left[-\left(\frac{\sigma_{x} h(\theta)}{\sigma_{e}}\right)^{n_{e}}\right]}+A\left(\sigma_{x}\right)^{n}[h(\theta)]^{n+1}
$$

The parameters in Equation (11) are listed in Table 2. The time-independent initial strains in Equation (1) under a constant stress $\sigma_{0}$ can be obtained as

$$
\varepsilon_{i n}=\frac{\sigma_{0}}{E_{0} \exp \left[-\left(\frac{\sigma_{0} h(\theta)}{\sigma_{e}}\right)^{n_{e}}\right]}+A\left(\sigma_{0}\right)^{n}[h(\theta)]^{n+1}
$$




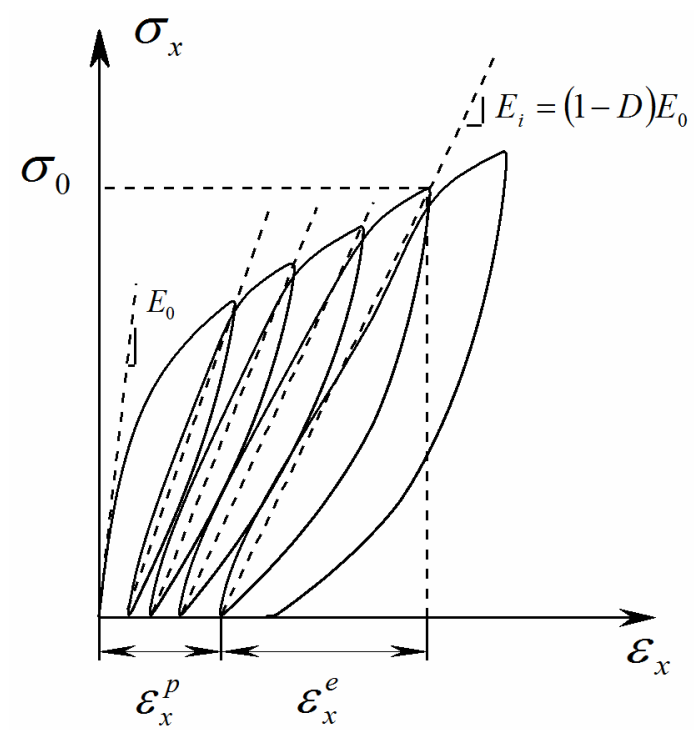

Figure 1. Cyclic loading-unloading tests for measuring damage variable and plastic strain [20].

In the present paper, the creep deformation is assumed to be time-dependent plastic deformation $\varepsilon_{p}(t)$, which is composed of time-dependent viscoelastic deformation and time-dependent viscous deformation. Sun and his coworkers [21] proposed a relationship between the plastic parameter $A$ and effective plastic strain rate $\overline{\dot{\varepsilon}}_{p}$ as

$$
A=\chi\left(\overline{\dot{\varepsilon}}_{p}\right)^{m}
$$

where $\chi$ and $m$ are material constants.

Thus, the effective stress-effective plastic strain relationship for quasi-UD composite can also be rewritten as

$$
\bar{\varepsilon}_{p}=\chi\left(\overline{\dot{\varepsilon}}_{p}\right)^{m}(\bar{\sigma})^{n}
$$

and

$$
\overline{\dot{\varepsilon}}_{p}=\frac{\dot{\varepsilon}_{p}}{h(\theta)}
$$

The differential equation is represented as

$$
\overline{\dot{\varepsilon}}_{p}=\left(\frac{1}{\chi}\right)^{\frac{1}{m}}(\bar{\sigma})^{\frac{-n}{m}}\left(\bar{\varepsilon}_{p}\right)^{\frac{1}{m}}
$$

Finally, the time-dependent strains $\varepsilon_{p}(t)$ at a constant loading stress $\sigma_{0}$ can be evaluated as

$$
\varepsilon_{p}(t)=\left[\frac{m-1}{m}\left(\frac{1}{\chi}\right)^{m}[h(\theta)]^{\frac{(1-n) /(m-1)}{m}}\left(\sigma_{0}\right)^{\frac{-n}{m}}\right]^{\frac{m}{m-1}} t^{\frac{m}{m-1}}
$$

Based on Equations (12) and (17), the sum of strains in Equation (1) can be rewritten as

$$
\begin{aligned}
\varepsilon_{t}=\frac{\sigma_{x}}{E_{0} \exp \left[-\left(\frac{\sigma_{x} h(\theta)}{\sigma_{e}}\right)^{n_{e}}\right]}+9.27 \times 10^{-8}\left(\sigma_{x}\right)^{4.5}(h(\theta))^{5.5} & \\
& \quad+\left[\frac{m-1}{m}\left(\frac{1}{\chi}\right)^{m}[h(\theta)]^{\frac{(1-n) /(m-1)}{m}}\left(\sigma_{0}\right)^{\frac{-n}{m}}\right]^{\frac{m}{m-1}} t^{\frac{m}{m-1}}
\end{aligned}
$$

The material constants in the model can be determined through cyclic loading-unloading tensile tests. Comparison with experimental results will be shown in Section 4. 


\section{Experiments}

\subsection{Materials and Samples}

The material systems investigated in the present study are quasi-UD E-glass/polypropylene composites. The reinforcement is quasi-UD E-glass fabric (as shown in Figure 2) provided by P-D Glasseiden $\mathrm{GmbH}$, Germany. It consists of $92 \%$ fibers in the warp direction and $8 \%$ of fibers in the weft direction. The matrix material is copolymer polypropylene (Moplen EP500V) supplied in granule form by LyondellBasell company, which is used to produce polypropylene film. The flat film of polypropylene was extruded using a single-screw extruder (Collin E30M; screw diameter $D=30 \mathrm{~mm}$, length-to-diameter ratio $L / D=25$, and the width of coat-hanger die $W=250 \mathrm{~mm}$ ) in combination with a chill-roll (Collin CR 136/350). The thickness and width of polypropylene film are about $0.2 \mathrm{~mm}$ and $210 \mathrm{~mm}$, respectively.

Quasi-UD E-glass/polypropylene composites were fabricated by means of hot compression from the stacked structure, which consists of three layers of polypropylene film and two layers of quasi-UD E-glass fabric. The thickness and fiber volume fraction of composite are $0.95 \pm 0.07 \mathrm{~mm}$ and $33.3 \%$ $\pm 0.5 \%$, respectively. Correspondingly, the fiber volume fraction in the warp and weft direction are approx. $30 \%$ and $3 \%$, respectively.
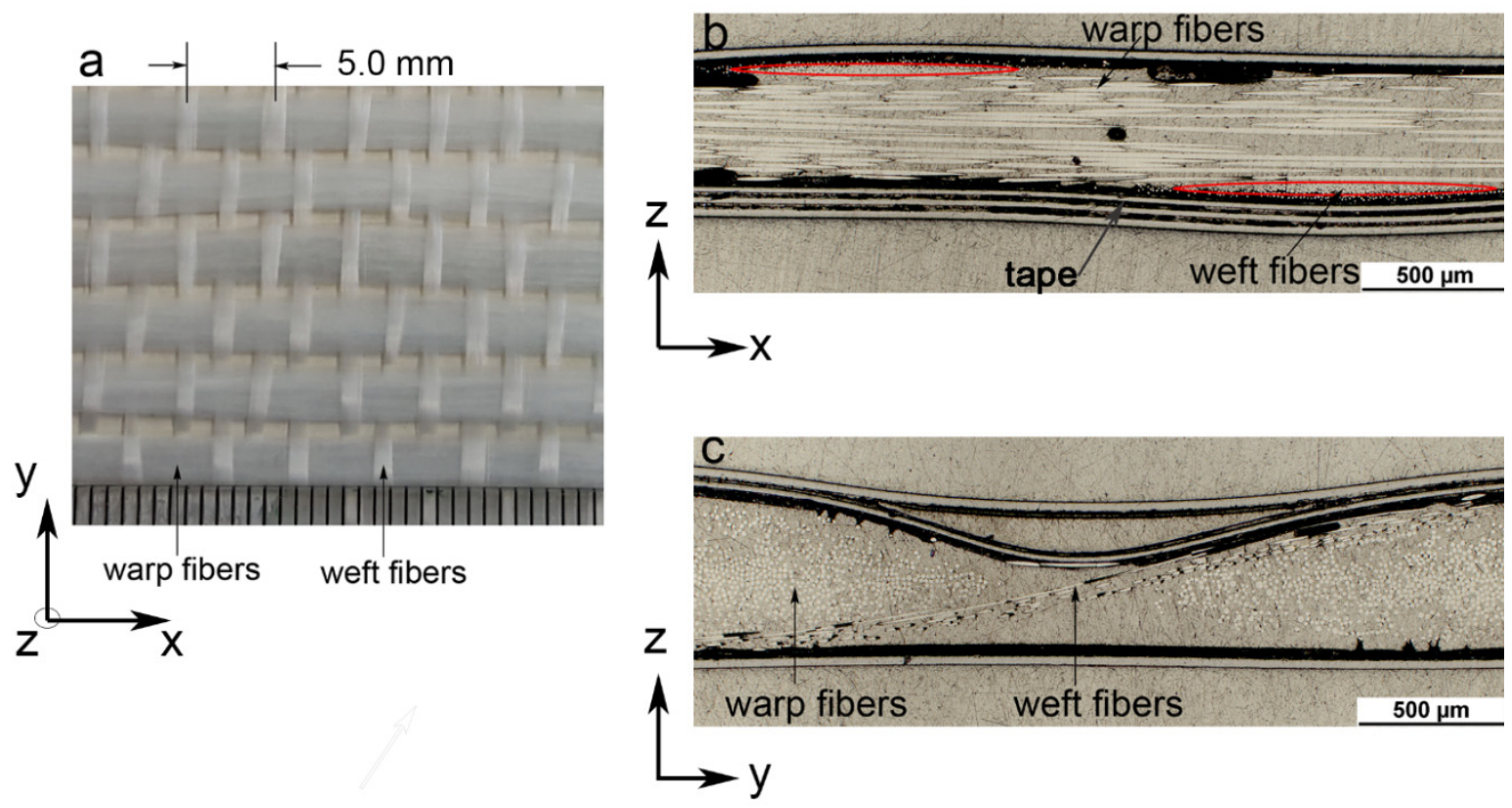

Figure 2. Quasi-UD E-glass fabric: (a) observed on the top sight (z direction), (b) cross-section of fabric along the warp fiber direction and (c) cross-section of fabric along the weft fiber direction.

Four kinds of off-axis samples $\left(\theta=10^{\circ}, 20^{\circ}, 45^{\circ}\right.$ and $\left.90^{\circ}\right)$ were cut from quasi-UD composites using a water-cooled sawing machine. The shape and dimensions of testing samples are according to Type 2 sample of ISO 527-4. The end tabs were attached on both ends of testing samples using the Loctite 406 instant adhesive and Loctite 770 Primer. More specifically, the conventional rectangular-shaped tabs were used for $90^{\circ}$ off-axis samples. In order to reduce the end-constrain effect induced by rigid clamps [25], the oblique end-tabs [26,27] were applied for $10^{\circ}, 20^{\circ}$ and $45^{\circ}$ off-axis samples.

\subsection{Experimental Procedure}

Tensile creep tests were carried on $10^{\circ}, 20^{\circ}$ and $90^{\circ}$ off-axis samples at room temperature. A testing machine of Zwick 1484 with a $100 \mathrm{KN}$ load was used. The extensometer with the gauge length of $50 \mathrm{~mm}$ was adopted to measure strains. The testing machine was set as the position control mode 
when the load reached the desired creep loading stress. In this initial stage, the loading speed of $1 \mathrm{~mm} / \mathrm{min}$ was used. After reaching the desired load, the testing machine was switched to the load control mode to maintain a constant loading stress. The duration of the creep test at each loading stress was $12 \mathrm{~h}$ if the sample did not damage before that. For each off-axis sample, the creep loading stress was set as $50 \%, 60 \%$ and $70 \%$ of its axial tensile strength, which were obtained from our previous work [20]. The details are listed in Table 1. In addition, to investigate damage accumulation effect on the creep strain, the elastic modulus of each testing sample after creep loading was compared with its initial elastic modulus before creep loading.

Table 1. Tensile creep testing plan for off-axis samples of quasi-UD E-glass/polypropylene composite.

\begin{tabular}{ccc}
\hline Off-Axis Sample & Creep Loading Stress (MPa) & Loading Time (h) \\
\hline $10^{\circ}$ & $20,25,30$ & \\
$20^{\circ}$ & $25,30,35$ & 12 \\
$90^{\circ}$ & $50,60,70$ & \\
\hline
\end{tabular}

To characterize the viscoplastic behavior of quasi-UD E-glass/polypropylene composite, cyclic loading-unloading tensile tests were performed on $10^{\circ}, 20^{\circ}, 45^{\circ}$ and $90^{\circ}$ off-axis samples at the loading speed of $1 \mathrm{~mm} / \mathrm{min}, 10 \mathrm{~mm} / \mathrm{min}$ and $100 \mathrm{~mm} / \mathrm{min}$. The gauge length is $50 \mathrm{~mm}$. Hence, the aforementioned crosshead speed corresponds approximately to the strain rate of $3.33 \times 10^{-4}$, $3.33 \times 10^{-3}$ and $3.33 \times 10^{-2} 1 / \mathrm{s}$, respectively. Off-axis samples were loaded and unloaded for four cycles with a gradually increasing peak stress level.

\section{Results and Discussion}

\subsection{Creep Behavior}

Figure $3 \mathrm{a}-\mathrm{c}$ shows the experimental strain-time curves for $10^{\circ}, 20^{\circ}$ and $90^{\circ}$ off-axis samples at three different loading levels $\left(0.5 \sigma_{x}, 0.6 \sigma_{x}, 0.7 \sigma_{x}\right)$. The creep strains for $90^{\circ}$ off-axis samples are inappreciable compared with the initial strains, regardless of stress level. The creep curves seem to get to a steady state with an almost constant creep strain rate after a transitional period. It is as expected since the plastic deformation is small when $90^{\circ}$ off-axis samples loaded under cyclic loading-unloading tests. Whereas, for $10^{\circ}$ and $20^{\circ}$ off-axis samples, the creep strains are appreciable compared to the initial strains. Moreover, the loading stress level has a significant influence on the creep strain rate of $10^{\circ}$ and $20^{\circ}$ off-axis samples.
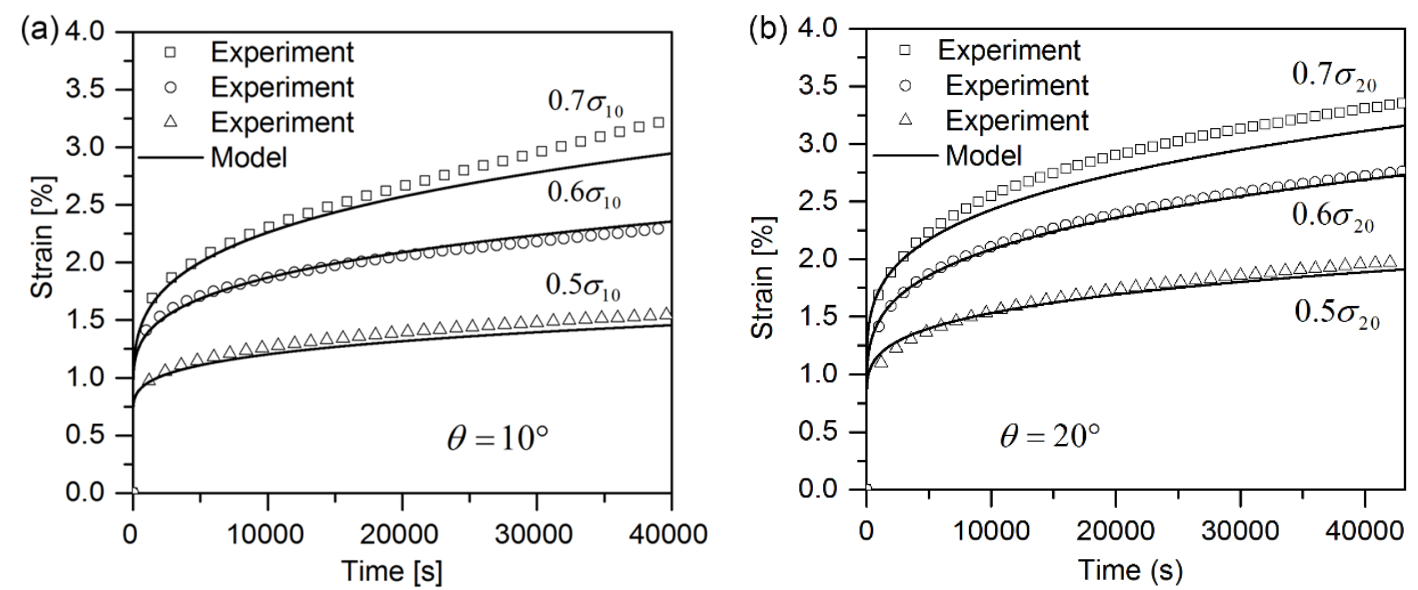

Figure 3. Cont. 


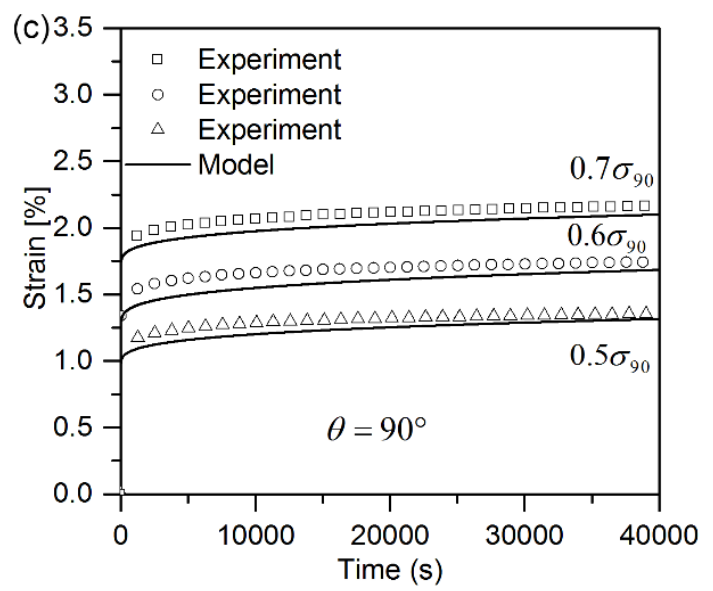

Figure 3. Strain-time curve for quasi-UD E-glass/polypropylene composite under off-axis loading with three creep loading levels: (a) $10^{\circ}$ off-axis samples; (b) $20^{\circ}$ off-axis samples and (c) $90^{\circ}$ off-axis samples.

The fracture surfaces of $90^{\circ}$ off-axis samples under quasi-static and creep loading were comparatively observed using a scanning electron microscopy (SEM), as shown in Figure 4 . For $90^{\circ}$ off-axis samples after quasi-static tensile loading, naked fibers and fibers covered with polypropylene can be observed in Figure 4a. Meanwhile, polypropylene was split along the transverse direction directly. These failure details indicate that the fracture modes belong to adhesive and matrix failure. On the other hand, $90^{\circ}$ off-axis samples under creep loading exhibit similar fracture behavior but more flexible/ductile behavior, as shown in Figure $4 \mathrm{~b}$.
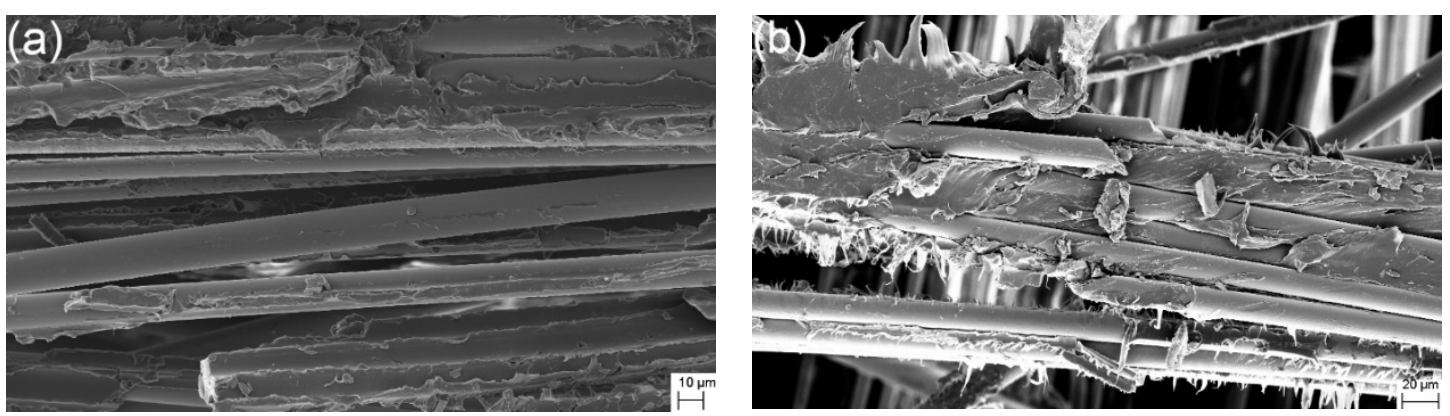

Figure 4. The fracture surface of $90^{\circ}$ off-axis samples: (a) quasi-static loading and (b) creep loading.

\subsection{Identification of Material Parameters for Creep Model}

Figure 5 gives representative cyclic loading-unloading curves of quasi-UD E-glass/polypropylene composite at different strain rates. One can notice that plastic strain (permeant strain) occurs in the off-axis samples after removing the applied stress for all the strain rates. Correspondingly, the experimental data of axial loading stress-axial plastic strain of composites at different strain rates can be directly achieved from cyclic loading-unloading tests. By means of Equations (7) and (8), the coalesced effective stress-effective plastic strains at different strain rates were determined with parameters $\boldsymbol{a}_{\mathbf{2}}$ and $\boldsymbol{b}_{\mathbf{2}}$ listed in Table 2, which are shown in Figure 6. As seen in Figure 6, the master effective stress-effective plastic strain curve for quasi-UD composite at different strain rates can be fitted with Equation (13) with the parameters $\boldsymbol{A}$ and $\boldsymbol{n}$ shown in Table 2. It can be found the parameter $\boldsymbol{n}$ is independent of strain rate.

Figure 7 shows the plastic parameter $\boldsymbol{A}$ in Equation (10) as a function of effective plastic strain rate for the off-axis samples of quasi-UD composite on a log-log scale. Furthermore, there is appreciable scatter in the data for the effective plastic rate. The fitting parameters $\chi$ and $m$ are listed in Table 3. 

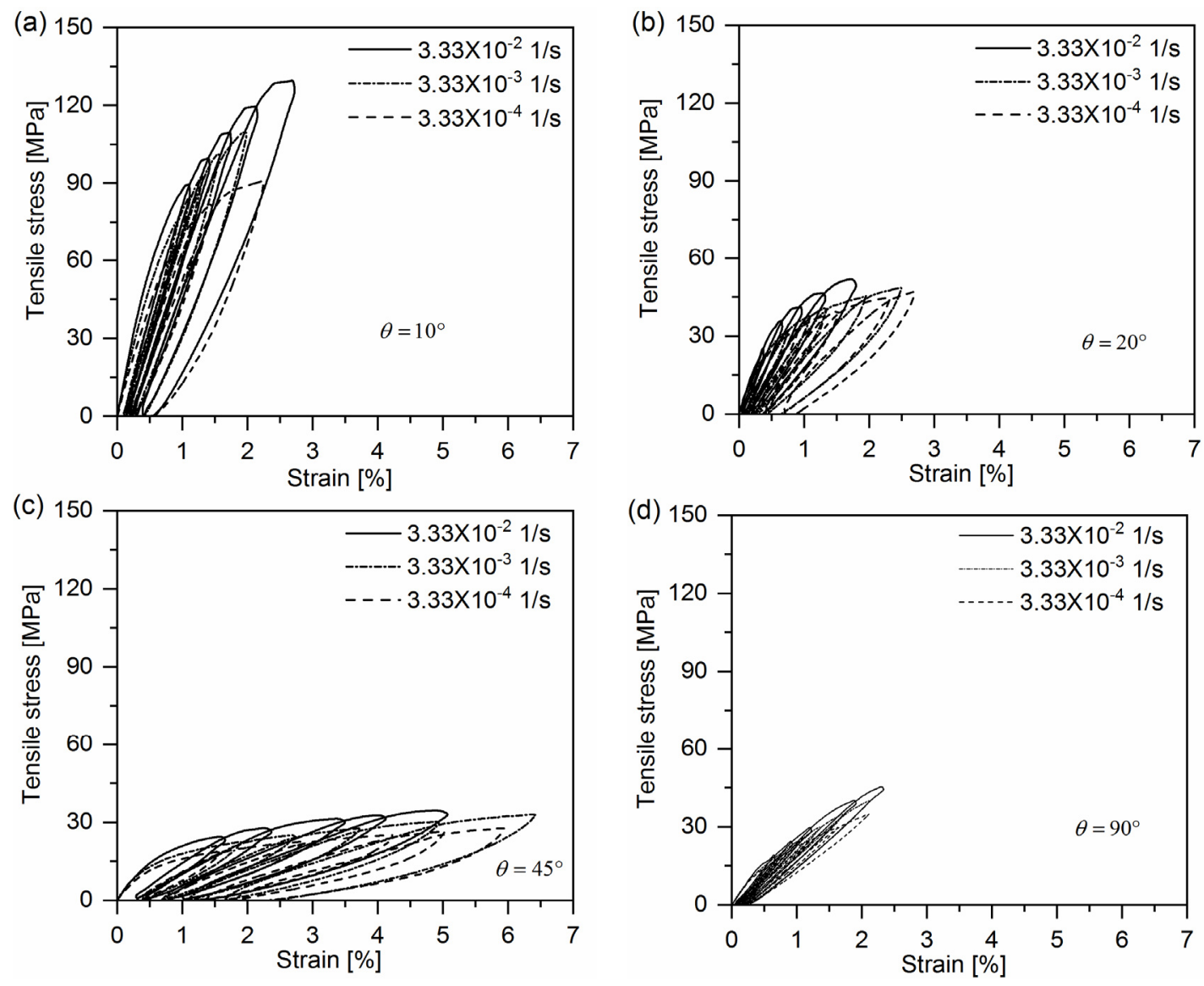

Figure 5. Representative cyclic loading-unloading curves for quasi-UD E-glass/polypropylene composite under different strain rates: (a) $\theta=10^{\circ}$; (b) $\theta=20^{\circ}$; (c) $\theta=45^{\circ}$ and (d) $\theta=90^{\circ}$.

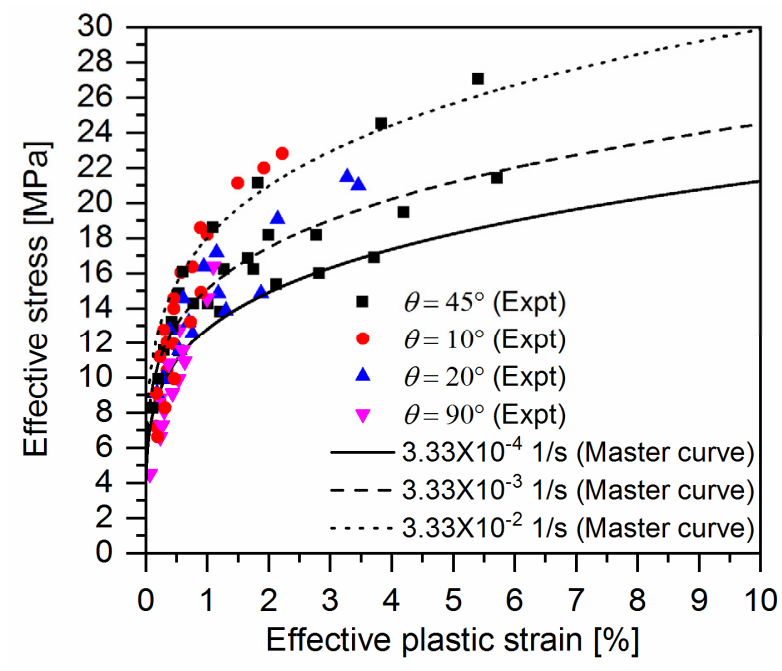

Figure 6. Effective stress-effective plastic curves for quasi-UD E-glass/polypropylene composite at different strain rates. 
Table 2. Plastic parameters for quasi-UD E-glass/PP composite at three different strain rates.

\begin{tabular}{|c|c|c|c|c|}
\hline \multirow{2}{*}{ Strain Rate (1/s) } & \multicolumn{2}{|c|}{ Material Constants (Equation (9)) } & \multicolumn{2}{|c|}{ Plastic Parameters (Equation (10) } \\
\hline & $a_{2}$ & $b_{2}$ & $A\left((\mathrm{MPa})^{-n}\right)$ & $n$ \\
\hline $3.33 \times 10^{-4}$ & & & $9.27 \times 10^{-8}$ & \\
\hline $3.33 \times 10^{-3}$ & 0.08 & 0.05 & $3.36 \times 10^{-8}$ & 4.5 \\
\hline $3.33 \times 10^{-2}$ & & & $1.96 \times 10^{-8}$ & \\
\hline
\end{tabular}

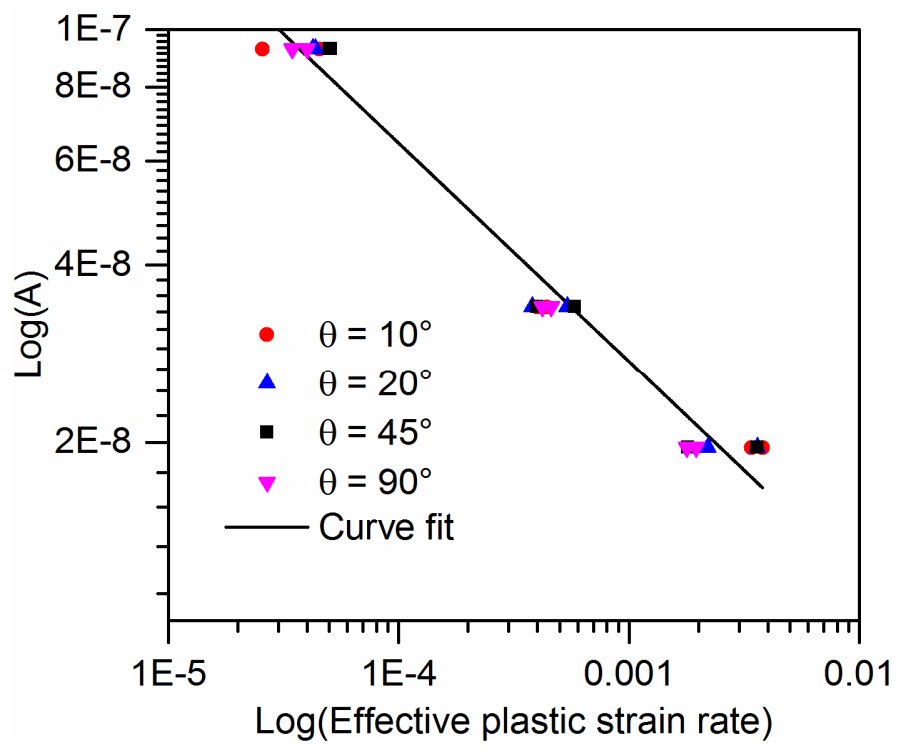

Figure 7. Plastic parameter $A$ for quasi-UD E-glass/polypropylene composite under different effective plastic strain rate.

Table 3. Material parameters for quasi-UD E-glass/polypropylene composite in Equation (14).

\begin{tabular}{cc}
\hline$x$ & -0.36 \\
\hline$m$ & -8.7 \\
\hline
\end{tabular}

\subsection{The Validation of Model}

Based on the material parameters listed in Tables 2 and 3 the strain-time curve of any off-axis sample of quasi-UD E-glass/polypropylene composite can be predicted by using Equation (18). The predicted results for $10^{\circ}, 20^{\circ}$ and $90^{\circ}$ off-axis samples are plotted in Figure 3. As shown in Figure 3, a satisfactory agreement between the predicted and experimental results is observed. However, for $90^{\circ}$ off-axis samples, Figure $3 \mathrm{c}$, the experimental results are higher than the predicted results from Equation (18) at three loading levels. Same phenomenon can be found in $10^{\circ}$ and $20^{\circ}$ off-axis sample at the loading level of $\mathbf{0 . 7} \sigma_{x}$, as shown in Figure $3 \mathrm{a}, \mathrm{b}$. It is supposed that the damage accumulations in the samples accelerated the creep strain rate, which is not taken into consideration for Equation (18). As shown in Figure 8, a large amount of damage events in the off-axis samples after tensile creep loading can be found. In addition, the damage factor $\left(=\frac{E_{c}}{E_{i}}\right)$ of off-axis samples under three different creep loading levels is shown in Figure 9, which reflects the reduction in the initial elastic modulus $E_{i}$ of off-axis samples after creep loading. As seen, the elastic modulus of off-axis samples $\left(\boldsymbol{E}_{\boldsymbol{c}}\right)$ after creep loading is lower compared to the initial elastic modulus $\left(E_{i}\right)$. Thus, the deviation between the experimental and predicted results in Figure 3 may be attributed to the damage accumulations in samples during creep loading. 


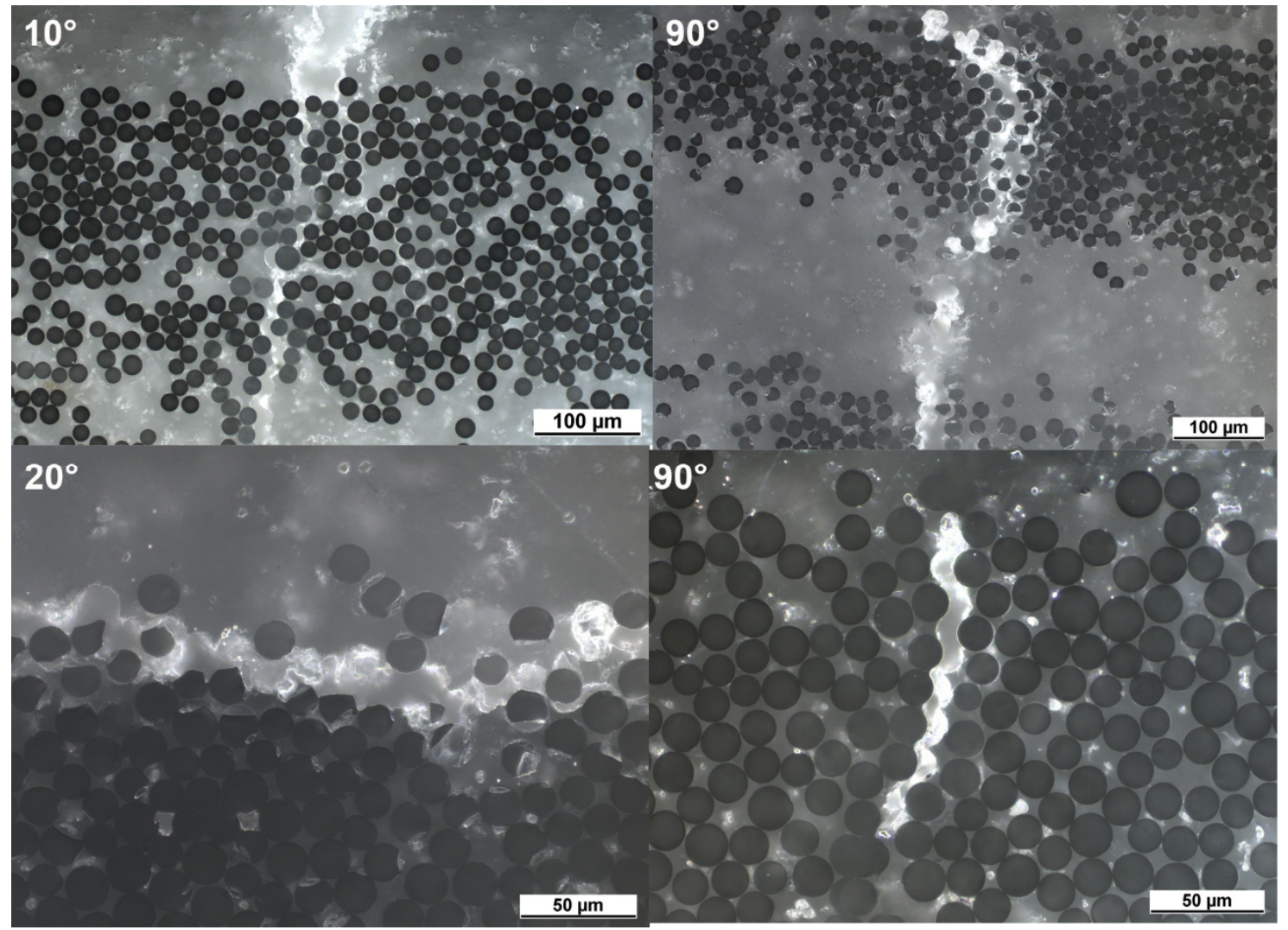

Figure 8. Damage events in off-axis samples after tensile creep loading.

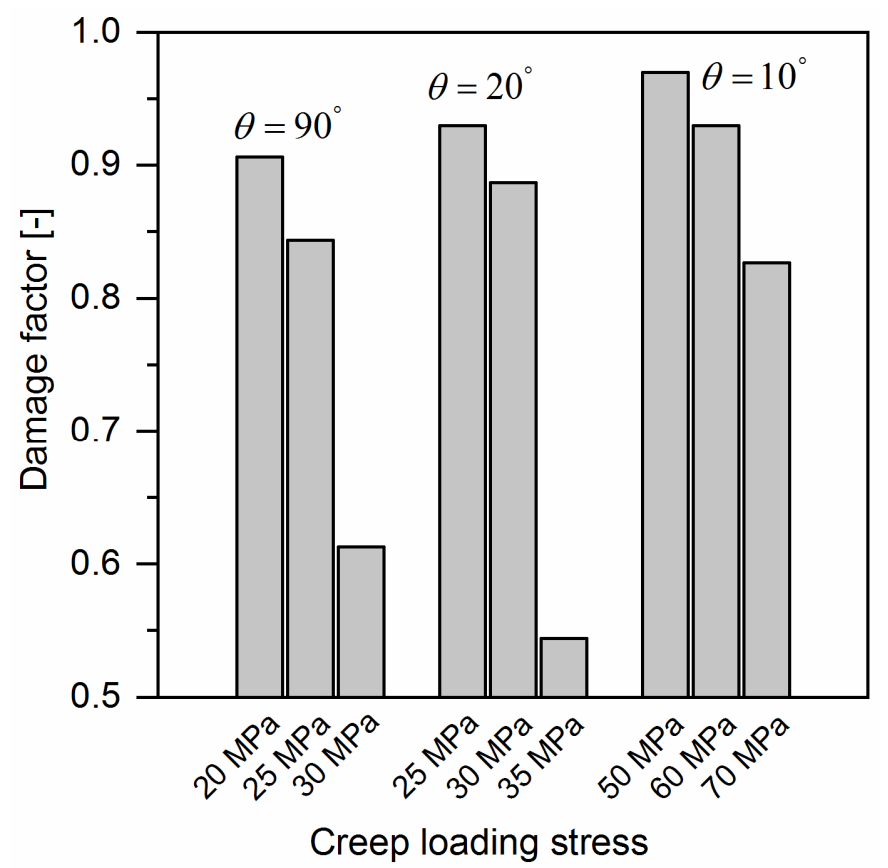

Figure 9. The comparison of elastic modulus of off-axis samples after creep loading with initial elastic modulus. 


\section{Conclusions}

The creep behavior of quasi-UD E-glass/polypropylene composites was investigated both experimentally and constitutively in this work. First of all, creep behavior was clearly observed for all the off-axis samples under a constant off-axis tensile loading. For $10^{\circ}$ and $20^{\circ}$ off-axis samples, the magnitude of creep strain became larger with increasing creep loading stress. The transient creep response was dominant in quasi-UD E-glass/polypropylene composite. The creep strain rate decreased with increasing creep strain. A phenomenological viscoplasticitiy model for quasi-UD E-glass/polypropylene composites was developed, in which the creep strain was assumed to be time-dependent plastic deformation. The material constants in the creep model were determined by using cyclic loading-unloading tensile tests. The predicted results agreed with the experimental results well. The minor deviation between the experimental and predicted creep behavior at high creep loading level may be attributed to the damage accumulations in the samples. Same failure mechanism was found in off-axis samples under quasi-static and creep loading.

Author Contributions: D.D. and Z.Z. conceived and designed the experiments. Z.Z. performed and analyzed the data. D.D. and B.J. helped with the revision of the paper.

Funding: This research received no external funding.

Acknowledgments: We acknowledge the support from DFG and Friedrich-Alexander-University Erlangen-Nürnberg (FAU) within the funding program Open Access Publishing. Zhanyu, Zhai also acknowledges the China Scholarship Council (CSC) for the financial support to her study at the University of Erlangen-Nuremburg.

Conflicts of Interest: There are no conflicts to declare.

\section{References}

1. Van den Oever, M.; Peijs, T. Continuous-glass-fibre-reinforced polypropylene composites II. Influence of maleic-anhydride modified polypropylene on fatigue behaviour. Compos. Part A Appl. Sci. Manuf. 1998, 29, 227-239. [CrossRef]

2. Brauner, C.; Herrmann, A.S.; Niemeier, P.M.; Schubert, K. Analysis of the non-linear load and temperature-dependent creep behaviour of thermoplastic composite materials. J. Thermoplast. Compos. Mater. 2017, 30, 302-317. [CrossRef]

3. Ehrenstein, G.W. Polymeric Materials: Structure, Properties, Applications; Carl Hanser Verlag GmbH Co KG: Munich, Germany, 2012.

4. Liou, W.; Tseng, C. Creep behavior of nylon-6 thermoplastic composites. Polym. Compos. 1997, 18, 492-499. [CrossRef]

5. Chung, I.; Sun, C.; Chang, I. Modeling creep in thermoplastic composites. J. Compos. Mater. 1993, 27, 1009-1029. [CrossRef]

6. Papanicolaou, G.; Zaoutsos, S. Viscoelastic constitutive modeling of creep and stress relaxation in polymers and polymer matrix composites. Creep Fatigue Polym. Matrix Compos. 2010, 1-47.

7. Pegoretti, A.; Ricco, T. Creep crack growth in a short glass fibres reinforced polypropylene composite. J. Mater. Sci. 2001, 36, 4637-4641. [CrossRef]

8. Robert, M.; Roy, R.; Benmokrane, B. Environmental effects on glass fiber reinforced polypropylene thermoplastic composite laminate for structural applications. Polym. Compos. 2010, 31, 604-611. [CrossRef]

9. Vas, L.M.; Bakonyi, P. Creep failure strain estimation of glass fibre/polypropylene composites based on short-term tests and Weibull characterisation. J. Reinf. Plast. Compos. 2013, 32, 34-41. [CrossRef]

10. Chevali, V.S.; Janowski, G.M. Flexural creep of long fiber-reinforced thermoplastic composites: Effect of processing-dependent fiber variables on creep response. Compos. Part A Appl. Sci. Manuf. 2010, 41, 1253-1262. [CrossRef]

11. Fliegener, S.; Hohe, J.; Gumbsch, P. The creep behavior of long fiber reinforced thermoplastics examined by microstructural simulations. Compos. Sci. Technol. 2016, 131, 1-11. [CrossRef]

12. Ropers, S.; Kardos, M.; Osswald, T.A. A thermo-viscoelastic approach for the characterization and modeling of the bending behavior of thermoplastic composites. Compos. Part A Appl. Sci. Manuf. 2016, 90, $22-32$. [CrossRef] 
13. Findley, W.; Lai, J.; Onaran, K.; Christensen, R. Creep and relaxation of nonlinear viscoelastic materials with an introduction to linear viscoelasticity. J. Appl. Mech. 1977, 44, 364. [CrossRef]

14. Gates, T.S.; Sun, C. Elastic/viscoplastic constitutive model for fiber reinforced thermoplastic composites. AIAA J. 1991, 29, 457-463. [CrossRef]

15. Guedes, R. Viscoplastic analysis of fiber reinforced polymer matrix composites under various loading conditions. Polym. Compos. 2009, 30, 1601-1610. [CrossRef]

16. Ogihara, S.; Moriwaki, S. Tensile creep deformation in unidirectional carbon/epoxy laminates under off-axis loading. J. Mater. Sci. 2004, 39, 3465-3467. [CrossRef]

17. Wang, C.; Sun, C. Experimental characterization of constitutive models for PEEK thermoplastic composite at heating stage during forming. J. Compos. Mater. 1997, 31, 1480-1506. [CrossRef]

18. Vasiukov, D.; Panier, S.; Hachemi, A. Non-linear material modeling of fiber-reinforced polymers based on coupled viscoelasticity-viscoplasticity with anisotropic continuous damage mechanics. Compos. Struct. 2015, 132, 527-535. [CrossRef]

19. Sun, C.; Chen, J. A simple flow rule for characterizing nonlinear behavior of fiber composites. J. Compos. Mater. 1989, 23, 1009-1020. [CrossRef]

20. Zhai, Z.; Jiang, B.; Drummer, D. Characterization of nonlinear response in quasi-unidirectional E-glass fabric reinforced polypropylene composites under off-axis tensile loading. Polym. Test. 2017, 63, 521-529. [CrossRef]

21. Thiruppukuzhi, S.V.; Sun, C. Models for the strain-rate-dependent behavior of polymer composites. Compos. Sci. Technol. 2001, 61,1-12. [CrossRef]

22. Ladeveze, P.; LeDantec, E. Damage modelling of the elementary ply for laminated composites. Compos. Sci. Technol. 1992, 43, 257-267. [CrossRef]

23. Cho, J.; Fenner, J.; Werner, B.; Daniel, I. A constitutive model for fiber-reinforced polymer composites. J. Compos. Mater. 2010, 44, 3133-3150. [CrossRef]

24. Zhai, Z.; Gröschel, C.; Drummer, D. Tensile behavior of quasi-unidirectional glass fiber/polypropylene composites at room and elevated temperatures. Polym. Test. 2016, 54, 126-133. [CrossRef]

25. Kawai, M.; Morishita, M.; Satoh, H.; Tomura, S.; Kemmochi, K. Effects of end-tab shape on strain field of unidirectional carbon/epoxy composite specimens subjected to off-axis tension. Compos. Part A Appl. Sci. Manuf. 1997, 28, 267-275. [CrossRef]

26. Sun, C.; Berreth, S. A new end tab design for off-axis tension test of composite materials. J. Compos. Mater. 1988, 22, 766-779. [CrossRef]

27. Sun, C.; Chung, I. An oblique end-tab design for testing off-axis composite specimens. Composites 1993, 24, 619-623. [CrossRef]

(C) 2018 by the authors. Licensee MDPI, Basel, Switzerland. This article is an open access article distributed under the terms and conditions of the Creative Commons Attribution (CC BY) license (http:/ / creativecommons.org/licenses/by/4.0/). 